\title{
Women's Midlife Health reviewer acknowledgement 2015
}

Siobán Harlow

\section{Contributing reviewers}

The editor of Women's Midlife Health would like to thank all of the reviewers who have contributed to the journal in Volume 1 (2015).

\section{Annemarie Koster \\ Netherlands}

Carrie Karvonen-Gutierrez

United States of America

\section{Cassandra Szoeke}

Australia

\section{Elsa Strotmeyer}

United States of America

Gail Greendale

United States of America

Gary Venolini

United States of America

Geena Athappilly

United States of America

Glinda Cooper

United States of America
Gloria Bachmann

United States of America

Janis Miller

United States of America

Jodi Flaws

United States of America

John Randolph

United States of America

Josefina Romaguera

Puerto Rico

Joyce Bromberger

United States of America

L. Elaine Waetjen

United States of America

Lorraine Dennerstein

Australia

\section{Martha Goetsch \\ United States of America}

Mary Sammel

United States of America

Michelle Warren

United States of America

Morgana Mongraw-Chaffin

United States of America

Nancy Phillips

United States of America

Nancy Woods

United States of America

Siobán Harlow

United States of America

Correspondence: wmhjournal@biomedcentral.com

University of Michigan, 500 S State St, Ann Arbor, MI 48109, USA 